\title{
Emerging and Converging Global Catastrophic BiOLOGICAL RisKS
}

\author{
Elizabeth E. Cameron
}

B IOLOGY HOLDS ENORMOUS PROMISE for enhancing human welfare in the 21 st century, including the potential to engineer and fabricate new life-extending treatments, vaccines, and therapeutics to combat disease; technologies to advance food security; and new sources of energy and materials. But the converse of this tremendous promise is the risk that an emerging or manufactured agent could spread quickly and kill millions, or hundreds of millions, before a countermeasure could be developed and dispensed. Equally important, such an event could rapidly shift global security dynamics by destabilizing economies, changing political landscapes, and disproportionately affecting specific populations. While the world continues to receive wake-up calls - including recent outbreaks of pandemic influenza, MERS, Ebola, and Zika-2 things are increasingly apparent. First, society is changing in ways that are likely to make a rapidly spreading biological agent even more deadly and destabilizing. Second, the world is largely unprepared for a fast-spreading, highly lethal, politically destabilizing biological event.

In starting a global dialogue about the definition and nature of global catastrophic biological risk (GCBR), the Center for Health Security provides a major service to the field. Within the proposed definition of GCBRs, the Center proposes to include "... events with world-reaching impacts on humanity, marked by profound and lasting social, economic, and political consequences, even if those events don't necessarily carry the potential to cause millions of fatalities [emphasis added]." The Center wisely avoids a narrow focus on the number of fatalities when it comes to GCBRs. The proposed definition appropriately recognizes the often unpredictable and far-reaching nature of GCBRs while affirming the potential interconnectivity of GCBRs with other types of global catastrophic risks (GCRs), such as climate change and nuclear war. For example, climate change can drive an increase in GCBRs and their global impact. And a GCBRparticularly an event with unclear origin, originating in an unstable region, or destabilizing a state possessing nuclear weapons-could exacerbate nuclear tension. In each case, the extent of global consequences, including the number of fatalities, will depend greatly on where a GCBR originates, how it spreads, and the ability of the international community to mount an effective, rapid response. The bottom line is that we need systems that prepare and practice for the worst potential outcomes, so that we can prevent them from occurring.

\section{The Risk}

GCBRs will continue to be magnified by global travel and trade, migration, terrorist interest in weapons of mass destruction, and rapid advances in technology that can create and manipulate pathogens with pandemic potential. Although progress has been made, the 2014-2016 Ebola epidemic highlighted the global risk to political and financial stability posed by weak health systems anywhere in the world.

In addition, while rapidly accelerating peaceful applications of biotechnology hold great promise for addressing emerging threats and must be pursued, these same technologies could also advance terrorist and state capability for developing and deploying biological weapons. Finally, escalating tension in unstable regions can further enhance the likelihood that a cross-border biological event could result in a catastrophic outcome. Senior officials must sustain a focus on GCBRs to thwart future world- and humanity-changing events.

(C) Elizabeth E. Cameron, 2017; Published by Mary Ann Liebert, Inc. This Open Access article is distributed under the terms of the Creative Commons Attribution Noncommercial License (http://creativecommons.org/licenses/by-nc/4.0/) which permits any noncommercial use, distribution, and reproduction in any medium, provided the original author(s) and the source are credited. 


\section{Today's Environment}

The global context is changing in ways that could increase the likelihood for epidemics to become GCBRs. Conflict is driving migration while also creating potential for new diseases to emerge and spread silently in regions of instability or ungoverned territories. Unchecked climate change will continue to exacerbate movements and will create new patterns of disease. And compounding all of these risks is the potential for terrorists to create, customize, and deploy biological agents-including within conflict-laden environments in which humanitarian assistance is challenging or impossible.

\section{We Are Not Prepared}

GCBRs quickly become local in an increasingly globalized world. Yet, the world is still unprepared-particularly if faced with an airborne pandemic with a high rate of spread and mortality. Despite intermittent focus and funding during periods of crisis, world leaders and policymakers have not established adequate capacity and financing mechanisms to prevent, detect, and rapidly respond to GCBRs. Ongoing challenges include: minimal global funding aimed at intentional or accidental GCBRs; no consensus among innovators or governments on norms and incentives for reducing biological risks associated with advancing technology; absence of adequate pandemic emergency funds; lack of threat awareness; dearth of creative financing mechanisms to spur sustained host country investment; and weak global biosurveillance, coupled with no global capability for pandemic prediction.

\section{Promising Signs, but Persistent Gaps}

New global initiatives are emerging, including the Coalition for Epidemic Preparedness Innovations (CEPI), the Global Health Security Agenda (GHSA), and the Joint External Evaluations (JEE). Taken together, these efforts focus on global capability to create and deliver new vaccines safely, affordably, and quickly (CEPI) and national preparedness and financing for countering biological threats (GHSA and JEE). However, additional creative mechanisms will be necessary to achieve the vision of a world safe and secure from GCBRs and to attain a global capability to prevent and counter disease threats that can keep pace with their emergence and the technology that can advance their creation.

\section{Vision}

It is in our collective interest for all countries to place GCBRs among their top national security priorities. Intense prevention efforts should be implemented so that emerging outbreaks don't become GCBRs in the first place. These activities must also minimize the potential for intentional misuse and accidental release so that the biological revolution, which is needed to save lives in the long run, does not itself cause a catastrophic outcome. Investments in global pandemic preparedness and the platforms necessary to manufacture, distribute, and dispense drugs and vaccines for any emerging threat will require a much greater degree of organization and synergistic financing among finance ministries, private sector actors, and donors. Governments, civil society, and the private sector should rightly view these investments as necessary down-payments-both to ensure that global efforts to address other transnational challenges are not thwarted by the impact of future GCBRs and to ensure that humanity is ready and able to reap the future rewards that modern biology promises.

Elizabeth E. Cameron, PhD Senior Director for Global Biological Policy and Programs Nuclear Threat Initiative 1210 E Street, $N E$ Washington, DC 20002

Email: cameron@nti.org 